\title{
SIMULAÇÃO DINÂMICA DO CRESCIMENTO DE BACTÉRIAS FLOCULANTES E FILAMENTOSAS NO TRATAMENTO DE EFLUENTES POR LODOS ATIVADOS: MODELO CONSIDERANDO ENRIJECIMENTO DOS FLOCOS
}

\author{
S. C. OLIVEIRA ${ }^{1}$ e R. P. FRANZOTE ${ }^{2}$ \\ ${ }^{1}$ Faculdade de Ciências Farmacêuticas, UNESP - Univ Estadual Paulista, Departamento de \\ Bioprocessos e Biotecnologia \\ E-mail: samueloliveira@fcfar.unesp.br \\ ${ }^{2}$ Consórcio Ductor-Encibra-Contécnica \\ E-mail: ricprado@hotmail.com
}

\begin{abstract}
RESUMO - Neste trabalho, a dinâmica de crescimento de bactérias floculantes e filamentosas em bioprocessos de tratamento de efluentes por lodos ativados é simulada para diferentes tempos de detenção hidráulica $\left(t_{h}\right)$. A um modelo matemático simples, baseado em expressões cinéticas do tipo Monod, foi introduzido um parâmetro relativo ao enrijecimento dos flocos, o qual estabilizou o modelo fazendo com que a coexistência estável das bactérias fosse prevista para um intervalo de valores de $t_{h}(4,08-22,22 \mathrm{~h})$ e não para um único valor como previsto pelo modelo original. Para o processo de lodos ativados operar eficientemente deve ocorrer certo equilíbrio entre o número de bactérias floculantes e filamentosas para que haja o enrijecimento filamentoso do lodo, evitando a sua lavagem. Deste modo, observou-se, através das simulações, que valores de $t_{h}$ em torno de $5,88 \mathrm{~h}$ parecem ser os mais adequados para a operação do processo.
\end{abstract}

\section{INTRODUÇÃO}

No âmbito do tratamento biológico de efluentes, existem vários processos de uso consagrado, como o processo de lodos ativados, o qual é amplamente utilizado, em nível mundial, para o tratamento de águas residuárias domésticas e industriais em que são necessários reduzidos requisitos de área e elevada qualidade do efluente tratado. No entanto, o processo de lodos ativados possui grau de mecanização superior ao de outros processos de tratamento, implicando uma operação mais sofisticada e um maior consumo energético.

Os flocos de lodos ativados constituem um amplo consórcio de microrganismos que interagem entre si segundo diversos tipos de relações (mutualismo, competição, inibição, comensalismo, etc.). Assim, a análise e a simulação de modelos matemáticos que descrevem a dinâmica populacional de espécies interagentes em processos de lodos ativados torna-se um requisito fundamental para a otimização e o controle de tais processos. 
Em um trabalho anterior, Oliveira e Franzote (2013) utilizaram um modelo matemático simples baseado em expressões cinéticas do tipo Monod para simular a dinâmica de crescimento de bactérias floculantes e filamentosas em bioprocessos de tratamento de efluentes por lodos ativados e analisar as condições operacionais que levam à coexistência estável de ambas as espécies de bactérias. Entretanto, o modelo apresentado não foi capaz de representar um conjunto de condições reais de operação para as quais se sabe ocorrer a coexistência de ambas as bactérias.

Deste modo, para obter um modelo mais realista, que represente a coexistência estável de ambas as bactérias, as equações do modelo devem ser modificadas para que isso seja possível. Essas modificações devem fazer com que o modelo descreva a coexistência das bactérias formadoras de flocos e as filamentosas no processo de tratamento por lodos ativados em um intervalo maior de condições operacionais, o que pode ser conseguido levando-se em conta o fenômeno de enrijecimento filamentoso do floco conforme proposto por Cenens et al. (2000).

A teoria do enrijecimento filamentoso dos flocos em sistemas de lodos ativados assume que a estrutura do floco é formada por dois níveis. O primeiro, chamado de microestrutura, depende da biofloculação das bactérias formadoras de flocos. O segundo é a macroestrutura, formada pelas bactérias filamentosas, as quais dão firmeza aos flocos, enquanto que as bactérias formadoras de flocos se agrupam com os polímeros extracelulares de maneira estável.

Neste trabalho, a dinâmica de crescimento de bactérias floculantes e filamentosas em processos de lodos ativados é simulada utilizando-se um modelo matemático que leva em consideração o fenômeno de enrijecimento dos flocos.

\section{MODELO MATEMÁTICO}

Baseado na teoria do enrijecimento filamentoso, o modelo original, apresentado em uma publicação anterior (Oliveira e Franzote, 2013), foi estendido para levar em conta as bactérias filamentosas que são utilizadas para a formação de flocos. Considerando o esquema mostrado na Figura 1 e admitindo não haver biomassa no efluente do sistema nem substrato na corrente de reciclo e nem na de descarte de biomassa, as equações de balanço de massa no reator e no sedimentador são dadas por:

$$
\begin{aligned}
& \frac{d C_{S}}{d t}=D\left(C_{S, \text { in }}-(1+r) C_{s}\right)-\frac{\mu_{f l o c} C_{X f l o c}}{Y_{f l o c}}-\frac{\mu_{f i l} C_{X f i l}}{Y_{f i l}} \\
& \frac{d C_{X f l o c}}{d t}=r D C_{X f l o c, s e d}-(1+r) D C_{X f l o c}+\mu_{f l o c} C_{X f l o c}+\alpha \frac{Y_{f l o c}}{Y_{f i l}} C_{X f i l}
\end{aligned}
$$


$\frac{d C_{X f i l}}{d t}=r D C_{X f i l, s e d}-(1+r) D C_{X f i l}+\mu_{f i l} C_{X f i l}-\alpha C_{X f i l}$

$\frac{d C_{S, \text { sed }}}{d t}=(1+r) \frac{Q}{V_{\text {sed }}} C_{S}-(1-w) \frac{Q}{V_{\text {sed }}} C_{S, \text { sed }}$

$\frac{d C_{X f l o c, s e d}}{d t}=(1+r) \frac{Q}{V_{\text {sed }}} C_{X f l o c}-(r+w) \frac{Q}{V_{\text {sed }}} \beta_{\text {floc }} C_{X f l o c}$

$\frac{d C_{X f i l, s e d}}{d t}=(1+r) \frac{Q}{V_{\text {sed }}} C_{X f i l}-(r+w) \frac{Q}{V_{\text {sed }}} \beta_{f i l} C_{X f i l}$

Considerando que a estabilidade e o comportamento do sistema são determinados pela dinâmica mais lenta, pode-se assumir que o sedimentador está sempre em estado estacionário em comparação com o reator. Assim:

$\frac{d C_{S, \text { sed }}}{d t}=0 \Rightarrow C_{S, \text { sed }}=\frac{(1+r)}{(1-w)} C_{S}$

$\frac{d C_{X f l o c, s e d}}{d t}=0 \Rightarrow C_{X f l o c, s e d}=\frac{(1+r)}{\beta_{f l o c}(w+r)} C_{X f l o c} \Rightarrow \frac{d C_{X f l o c}}{d t}=\mu_{f l o c} C_{X f l o c}-\bar{D} C_{X f l o c}+\alpha \frac{Y_{f l o c}}{Y_{f i l}} C_{X f i l}$

$\frac{d C_{X f i l, \text { sed }}}{d t}=0 \Rightarrow C_{X f i l, s e d}=\frac{(1+r)}{\beta_{f i l}(w+r)} C_{X f i l} \Rightarrow \frac{d C_{X f i l}}{d t}=\mu_{f i l} C_{X f i l}-\bar{D} C_{X f i l}-\alpha C_{X f i l}$

onde: $\bar{D}=\frac{w(1+r)}{r+w} D$

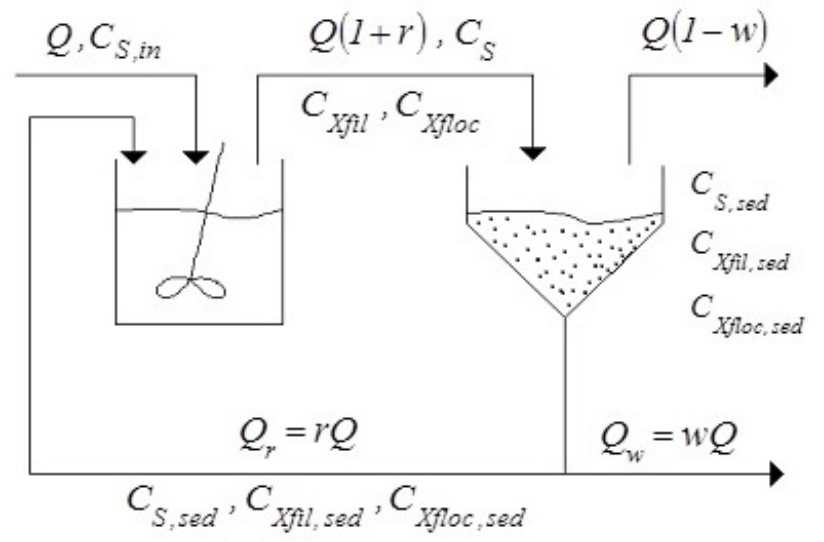

Figura 1 - Esquema do processo de lodos ativados para fins de modelagem matemática. 
Nestas equações, o parâmetro $\alpha\left(\mathrm{h}^{-1}\right)$, do ponto de vista biológico, representa a fração de bactérias filamentosas incorporadas ao floco enquanto que, do ponto de vista matemático, representa um fator estabilizante do modelo, o qual faz com que a coexistência estável das bactérias ocorra em um intervalo de valores de $\bar{D}\left(\bar{D}_{1}<\bar{D}<\bar{D}_{2}\right)$ e não em um único valor como ocorre para o modelo original (Figura 2).
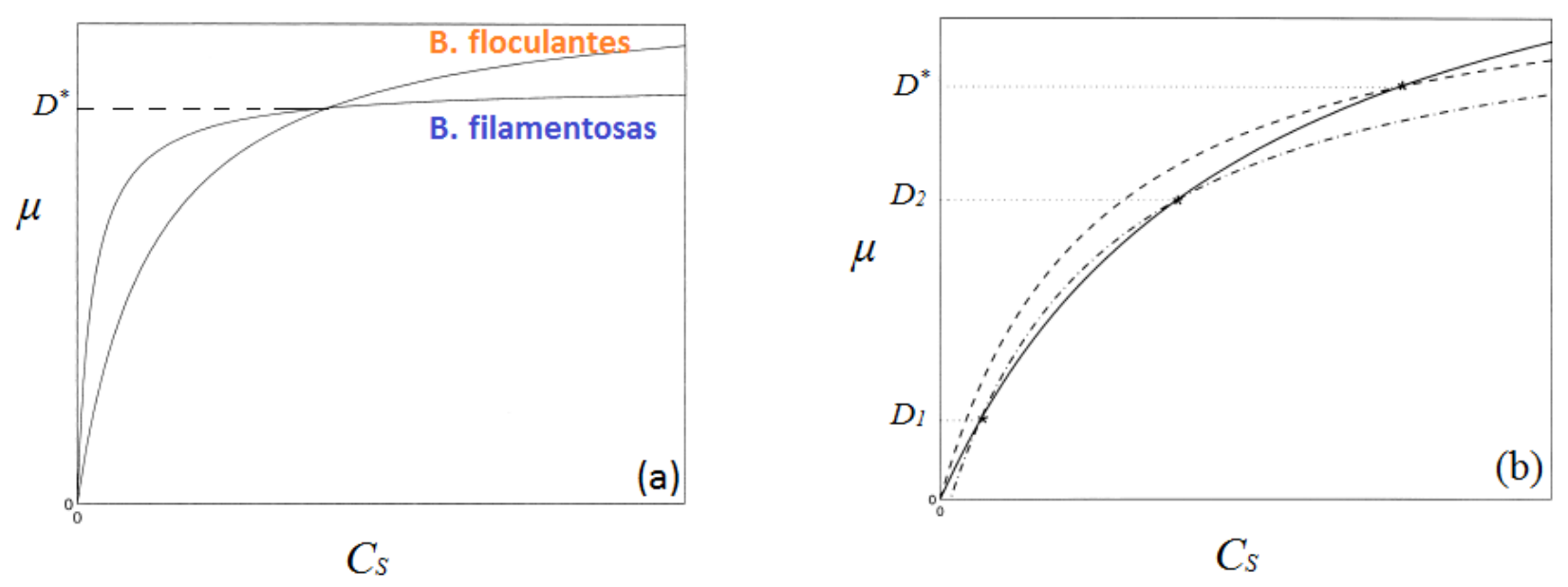

Figura 2 - Comportamento qualitativo da taxa específica de crescimento das bactérias floculantes e filamentosas quando $K_{S, \text { floc }}>K_{S, \text { fil }}$ e $\mu_{\text {max ffloc }}>\mu_{\text {max fil }}$ : (a) - modelo original ; (b) - modelo modificado

As expressões matemáticas para os valores de $\bar{D}$ que definem o intervalo para o qual a coexistência das bactérias ocorre são as seguintes (Cenens et al., 2000) :

$\bar{D}_{1}=\frac{\left(\bar{D}^{*}-\alpha\right)-\sqrt{\left(\alpha-\bar{D}^{*}\right)^{2}-\frac{4 \alpha K_{S, \text { fil }} \mu_{\text {max }, \text { floc }}}{K_{S, \text { floc }} K_{S, \text { fil }}}}}{2}$

$\bar{D}_{2}=\frac{\left(\bar{D}^{*}-\alpha\right)+\sqrt{\left(\alpha-\bar{D}^{*}\right)^{2}-\frac{4 \alpha K_{S, \text { fil }} \mu_{\text {max }, \text { floc }}}{K_{S, \text { floc }}-K_{S, f i l}}}}{2}$

$\bar{D}^{*}=\frac{\mu_{\max , f i l} K_{S, \text { floc }}-\mu_{\max , \text { floc }} K_{S, \text { fil }}}{K_{S, \text { floc }}-K_{S, \text { fil }}}$

Na Tabela 1 estão apresentados os valores dos parâmetros cinéticos e operacionais utilizados na simulação do processo de lodos ativados. 
Tabela 1 - Parâmetros usados na simulação do processo de lodos ativados

\begin{tabular}{|l|c|c|c|c|c|c|}
\hline Bactérias & $\begin{array}{c}\boldsymbol{\mu}_{\max } \\
\left(\mathbf{h}^{-\mathbf{1}}\right)\end{array}$ & $\begin{array}{c}\boldsymbol{K}_{S} \\
(\mathbf{g} / \mathbf{L})\end{array}$ & $\begin{array}{c}Y \\
(\mathbf{g} / \mathbf{g})\end{array}$ & \multicolumn{3}{|l|}{ Parâmetros operacionais } \\
\hline Floculantes & 1,1 & 0,4 & 0,6 & $r(-)$ & $w(-)$ & $C_{S, \text { in }}(\mathrm{g} / \mathrm{L})$ \\
& & & & & & 25,0 \\
\hline Filamentosas & 0,7 & 0,2 & 0,6 & 0,5 & 0,1 & \\
\hline
\end{tabular}

\section{RESULTADOS E DISCUSSÃO}

Os valores de $\bar{D}_{1}$ e $\bar{D}_{2}$ são completamente determinados pelos parâmetros cinéticos e também pelo valor de $\alpha$. Por meio de diversas simulações, verificou-se numericamente que o valor de $\alpha$ deve estar compreendido entre 0 e $0,0180652 \mathrm{~h}^{-1}$ para que os valores de $\bar{D}_{1}$ e $\bar{D}_{2}$ sejam números reais positivos. Valores de $\alpha$ acima do limite superior levam à obtenção de números complexos para $\bar{D}_{1}$ e $\bar{D}_{2}$.

Estes valores de $\bar{D}_{1}$ e $\bar{D}_{2}$ levam a três casos distintos, os quais estão sumarizados na Tabela 2. Para a simulação desses casos, os valores dos parâmetros usados foram os mesmos da Tabela 1, além de $\alpha=0,01 \mathrm{~h}^{-1}$ e as seguintes condições iniciais: $C_{S}(0)=1 \mathrm{~g} / \mathrm{L}, C_{f l o c}(0)=10 \mathrm{~g} / \mathrm{L}$ e $C_{f i l}(0)=10 \mathrm{~g} / \mathrm{L}$. Assim, simulando-se a interação das bactérias floculantes com as filamentosas utilizando-se o modelo modificado, obtém-se a Figura 3 para os casos descritos na Tabela 2.

Para o modelo do processo de lodos ativados considerando o enrijecimento filamentoso, as condições iniciais impostas e os parâmetros cinéticos adotados, observa-se que ambas as espécies coexistem no intervalo de $\bar{D}$ entre 0,045 e $0,245 \mathrm{~h}^{-1}$ (tempo de detenção hidráulica $t_{h}$ entre 22,22 e $4,08 \mathrm{~h}$ ), permitindo, deste modo, uma maior flexibilidade das condições operacionais do sistema de tratamento (Figura 4).

Apesar de as bactérias filamentosas competirem com as floculantes pelo mesmo substrato, elas são importantes para o bom andamento do processo. Assim, para o processo de lodos ativados operar eficientemente deve ocorrer certo equilíbrio entre o número de bactérias formadoras de flocos e o de filamentosas para que haja o enrijecimento filamentoso do lodo não permitindo a sua lavagem. Deste modo, valores de $\bar{D}$ em torno de $0,17 \mathrm{~h}^{-1}$, ou seja, $t_{\mathrm{h}}$ em torno de $5,88 \mathrm{~h}$ parecem ser os mais adequados para a operação do processo. 
Tabela 2 - Estados estacionários e condições de estabilidade para o processo de lodos ativados segundo o modelo modificado

\begin{tabular}{|c|c|c|}
\hline & $\begin{array}{l}\text { Valores calculados } \\
\text { analiticamente }\end{array}$ & $\begin{array}{l}\text { Valores calculados } \\
\text { numericamente }\end{array}$ \\
\hline $\begin{array}{l}\text { 1. Lavagem das bactérias filamentosas } \\
\text { i. Valores no estado estacionário: } \\
\bar{D}=\mu_{f l o c} \\
C_{S}^{*}=\frac{K_{S, f l o c} \bar{D}}{\mu_{\max , f l o c}-\bar{D}} \\
C_{X f l o c}^{*}=\left(\frac{C_{S, i n}}{1+r}-C_{S}^{*}\right) Y_{f l o c} \frac{r+w}{w} \\
C_{X f i l}^{*}=0 \\
\text { ii. Estabilidade: } \\
\text { Estável se } \bar{D}<\bar{D}_{1} \vee \bar{D}>\bar{D}_{2}\end{array}$ & $\begin{array}{l}\bar{D}_{1}=0,0448750891 \\
\bar{D}_{2}=0,245124862 \\
\bar{D}=0,5 \\
C_{S}^{*}=0,333333272 \\
C_{X f l o c}^{*}=58,8 \\
C_{X f i l}^{*}=0\end{array}$ & $\begin{array}{l}\bar{D}=0,5 \\
C_{S}^{*}=0,333333333 \\
C_{X f l o c}^{*}=58,8 \\
C_{X f i l}^{*}=3,26517377 \times 10^{-314}\end{array}$ \\
\hline $\begin{array}{l}\text { 2. Coexistência } \\
\text { i. Valores no estado estacionário: } \\
\bar{D}=\mu_{f i l}-\alpha \\
C_{S}^{*}=\frac{K_{S, f i l}(\bar{D}+\alpha)}{\mu_{\max , f i l}-(\bar{D}+\alpha)}\end{array}$ & $\begin{array}{l}\bar{D}=0,1 \\
C_{S}^{*}=0,037288135 \\
C_{X f l o c}^{*}=36,95063512 \\
C_{X f i l}^{*}=22,91512816\end{array}$ & $\begin{array}{l}\bar{D}=0,1 \\
C_{S}^{*}=0,0372881356 \\
C_{X f l o c}^{*}=36,9506382 \\
C_{X f i l}^{*}=22,9151245\end{array}$ \\
\hline $\begin{array}{l}C_{X f l o c}^{*}=\left(\frac{C_{S, i n}}{1+r}-C_{S}^{*}\right) \frac{\alpha Y_{f l o c}}{\alpha+\bar{D}-\mu_{f l o c}} \frac{w+r}{w} \\
C_{X f l i}^{*}=\left(\frac{C_{S, i n}}{1+r}-C_{S}^{*}\right) \frac{Y_{f i l}\left(\bar{D}-\mu_{f l o c}\right)}{\alpha+\bar{D}-\mu_{f l o c}} \frac{w+r}{w} \\
\text { ii. Estabilidade: Estável se } D_{1}<\bar{D}<D_{2}\end{array}$ & & \\
\hline $\begin{array}{l}\text { 3. Lavagem das bactérias floculantes e } \\
\text { filamentosas } \\
\text { i. Valores no estado estacionário: } \\
C_{S}^{*}=\frac{C_{S, i n}}{1+r} \\
C_{X f l o c}^{*}=0 \\
C_{X f i l}^{*}=0 \\
\text { ii. Estabilidade: } \\
\text { Estável se } \bar{D}>\mu_{\max , f l o c} \frac{C_{S, i n}}{K_{S, f l o c}+C_{S, i n}}\end{array}$ & $\begin{array}{l}C_{S}^{*}=16,6666667 \\
C_{X f l o c}^{*}=0 \\
C_{X f i l}^{*}=0\end{array}$ & $\begin{array}{l}C_{S}^{*}=16,6666667 \\
C_{X f l o c}^{*}=2,00343619 \times 10^{-320} \\
C_{X f i l}^{*}=4,76773348 \times 10^{-321}\end{array}$ \\
\hline
\end{tabular}



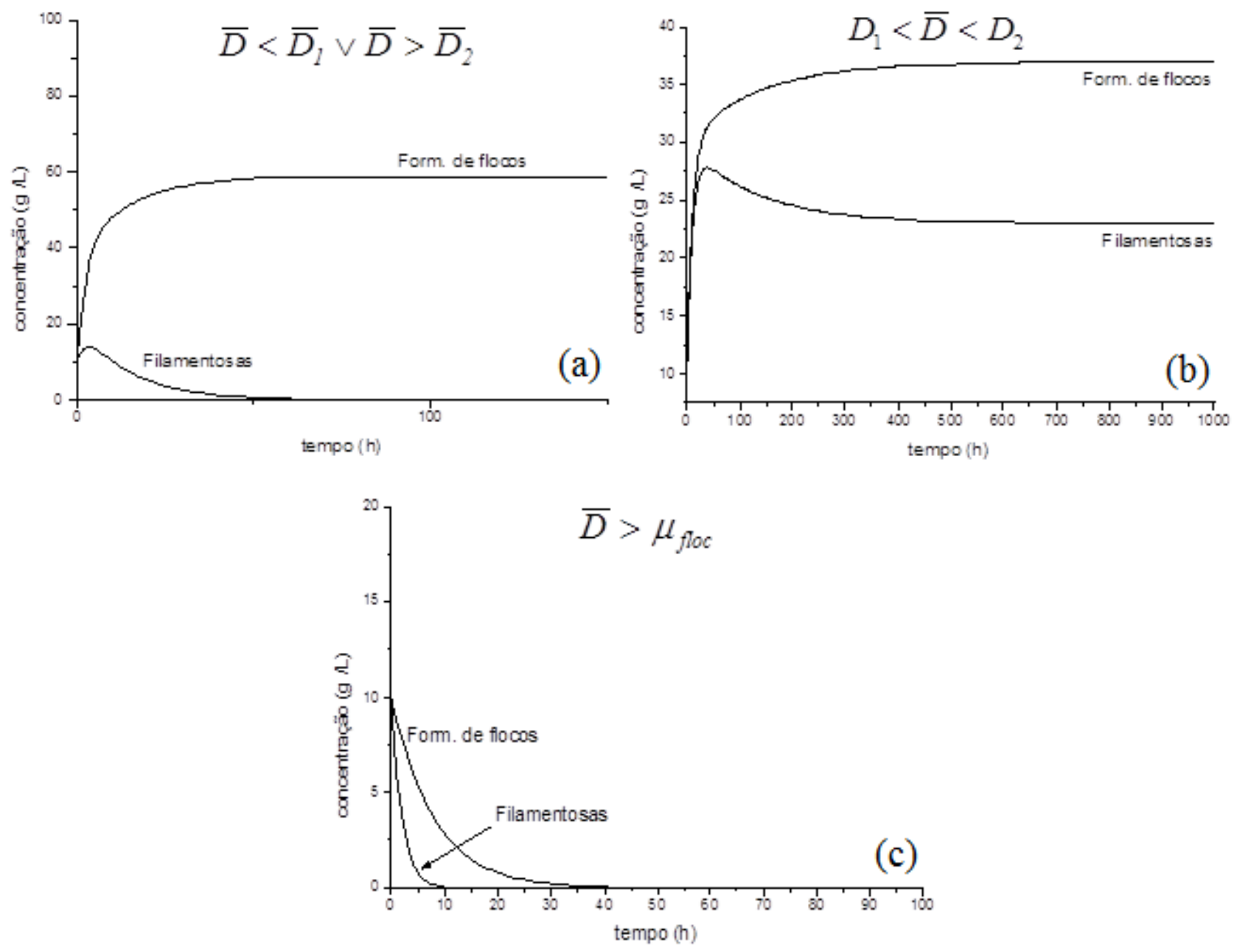

Figura 3 - Dinâmica de crescimento de bactérias floculantes e filamentosas em um processo de lodos ativados: (a) Extinção das bactérias filamentosas ; (b) Coexistência estável ; (c) Extinção de ambas as bactérias.

\section{CONCLUSÕES}

A partir de um modelo matemático que considera o fenômeno de enrijecimento filamentoso dos flocos foi possível prever, para um amplo intervalo de $t_{h}(4,08-22,22 \mathrm{~h})$, a coexistência estável de bactérias floculantes e filamentosas em um processo de tratamento de efluentes por lodos ativados. A simulação do modelo dentro do intervalo de $t_{h}$ especificado revelou que, para um tempo de detenção hidráulica em torno de 6 horas, ocorre a coexistência das bactérias em quantidades aproximadamente iguais, condição essencial para uma boa eficiência do processo. Este tempo de detenção de 6 horas, para o qual a coexistência ocorre, aproxima-se bem do tempo usualmente praticado em processos de lodos ativados convencionais. 

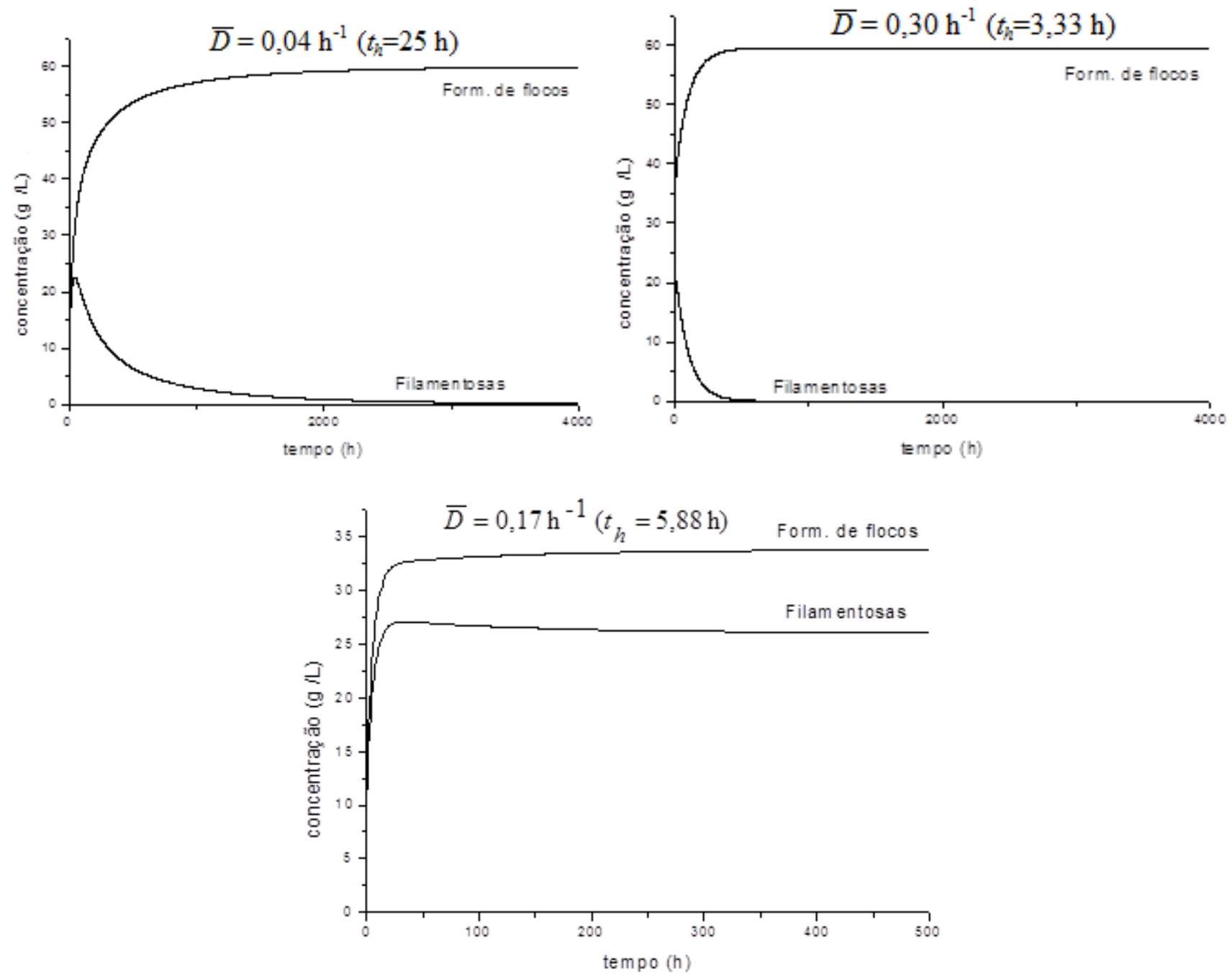

Figura 4 - Simulação da dinâmica de crescimento de bactérias floculantes e filamentosas em um processo de lodos ativados para diferentes condições operacionais.

\section{REFERÊNCIAS}

OLIVEIRA, S. C.; FRANZOTE, R. P. Modelagem matemática e simulação da dinâmica de crescimento de bactérias floculantes e filamentosas em bioprocessos de tratamento de efluentes por lodos ativados. In: Anais do XIX Simpósio Nacional de Bioprocessos/X Simpósio de Hidrólise Enzimática de Biomassas. Foz do Iguaçu-PR, 2013.

CENENS, C.; SMETS, I. Y.; VAN IMPE, J. F. Modeling the Competition Between Floc-Forming and Filamentous Bacteria in Activated Sludge Waste Water Treatment Systems - II: A Prototype Mathematical Model Based on Kinetic Selection and Filamentous Backbone Theory. Wat. Res. v. 34 (9), p. 2535-2541, 2000. 